\title{
IDENTIFICAÇÃO DE LINHAGENS AVANÇADAS DE ALFACE QUANTO À RESISTÊNCIA A Meloidogyne javanica
}

\author{
Identification of advanced lineages of lettuce resistant to Meloidogyne javanica
}

\author{
Sindynara Ferreira ${ }^{1}$, Vitória Lara França Vieira ${ }^{2}$, Luiz Antonio Augusto Gomes ${ }^{2}$, \\ Wilson Roberto Maluf², José Luiz Sandes de Carvalho Filho ${ }^{3}$
}

\begin{abstract}
RESUMO
Objetivou-se, no presente trabalho, avaliar o comportamento de linhagens de alface quanto à resistência ao nematoide das galhas Meloidogyne javanica, identificando a provável existência de linhagens homozigotas para o caráter. Foram utilizados como tratamentos cinco linhagens $\mathrm{F}_{4}$ e seis linhagens $\mathrm{F}_{5}$, juntamente com as testemunhas 'Grand Rapids' (resistente) e 'Regina 71' (suscetível), além da cultivar de tomateiro Santa Clara. A semeadura foi feita em bandejas de poliestireno expandido, sendo o substrato infestado com ovos de $M$. javanica, na proporção de 30 ovos $\mathrm{cm}^{-3}$ de substrato. Aos quarenta e sete dias após a infestação, cada planta foi avaliada individualmente quanto aos seguintes caracteres: índice visual de galhas, número de ovos, fator de reprodução e índice de reprodução. Cada linhagem foi comparada com as testemunhas 'Regina 71' e 'Grand Rapids', para cada característica, através do teste de Dunnett (5\%), obtendo-se a significância em relação a cada uma das testemunhas, permitindo a classificação de cada linhagem como homozigota resistente, homozigota suscetível ou segregante. Pelos resultados obtidos conclui-se que seis linhagens (AFX $018 \mathrm{C} 002$ 23 15, AFX 018C 00223 22, AFX 018C 00542 20, AFX 018C 00544 22, AFX 018C 0054428 e AFX $022 B 010$ 31) foram consideradas homozigotas resistentes para as características avaliadas, podendo ser utilizadas como novas fontes de resistência ao $M$. javanica em programas de melhoramento de alface.
\end{abstract}

Termos para indexação: Lactuca sativa L., melhoramento, seleção.

\begin{abstract}
The objective of the present work was to evaluate the behavior of lettuce lineages as for resistence to nematode from the Meloidogyne javanica root-knots, identifying probable existence of homozigote lineages for the characteristics. Five $\mathrm{F}_{4}$ and six $\mathrm{F}_{5}$ lineages were used as treatments together with the controls 'Grand Rapids' (resistent) and 'Regina 71' (susceptible), apart from the Santa Clara tomato plant cultivar. The sowing was done over expanded polystyrene trays with the substrate infested by $M$. javanica eggs in the proportion of 30 eggs $\mathrm{cm}^{-3}$ of substrat. At 47 days after infestation, each plant was evaluated individually as for the following characteristics: visual level of root-knots, number of eggs, level and factor of reproduction. Every lineage was compared to the 'Regina 71' and 'Grand Rapids' for each characteristic, through Dunnett (5\%) test, obtaining the significance in relation to each control, allowing the classification of each lineage as resistant homozigote, susceptible homozigote or segregated. The results obtained showed that six lineages (AFX 018C 00223 15, AFX 018C 00223 22, AFX 018C 00542 20, AFX 018C 00544 22, AFX $018 \mathrm{C} 005$ 4428 and AFX 022B 010 31) were considered resistant homozigote for the evaluated characteristics, and maybe being used as new sources of resistance to $M$. javanica in lettuce breeding program.
\end{abstract}

Index terms: Lactuca sativa L., breeding, selection.

(Recebido em 16 de junho de 2009 e aprovado em 27 de abril de 2010)

\section{INTRODUÇÃO}

A alface (Lactuca sativa L.) é a hortaliça folhosa de maior importância econômica para o Brasil, sendo consumida in natura na forma de salada. A produção comercial de alface, em condições de elevadas temperaturas, tem sido afetada por nematoides pertencentes ao gênero Meloidogyne, especialmente M. incognita e M. javanica (Fiorini et al., 2007; Silva et al., 2008).
As áreas plantadas com hortaliças são normalmente submetidas a cultivos intensivos durante todo o ano, podendo ser grandes os danos causados por fitonematoides (Campos, 1985). Esses patógenos apresentam alta taxa reprodutiva, acumulando no solo grande população de ovos (Campos et al., 2001). Os nematoides que atacam raízes geralmente provocam os mesmos sintomas de deficiência mineral, já que a absorção de nutrientes é dificultada em razão dos danos no sistema radicular (Lopes \& Santos, 1994), ocasionando,

${ }^{1}$ Instituto Federal de Educação, Ciência e Tecnologia do Sul de Minas Gerais/IFSULDEMINAS - Praça Tiradentes - 416 - 37576-000 - Inconfidentes, MG sindynaraferreira@yahoo.com.br

${ }^{2}$ Universidade Federal de Lavras/UFLA - Departamento de Agricultura/DAG - Lavras, MG

${ }^{3}$ Universidade Federal Rural de Pernambuco/UFRPER - Departamento de Agricultura - Recife, PE 
principalmente, amarelecimento da plantas, tamanho reduzido e murcha da parte aérea.

As perdas causadas por esse patógeno são variáveis e dependentes de sua densidade populacional, da suscetibilidade do hospedeiro, das condições ambientais, da interação com outros patógenos e de condições fisiológicas da planta (Santos \& Souza, 1996).

Os fitonematoides são de difícil controle, pois geralmente ficam no solo ou no interior de raízes ou outros órgãos das plantas. Alguns possuem estrutura de resistência, outros entram em dormência e outros se reproduzem rapidamente e em grande número (Freitas, 2003). Normalmente, tenta-se fazer o controle desse patógeno por meio de práticas culturais como revolvimento do solo, irrigação após revolvimento, pousio, solarização, inundação, adubação verde, rotação de cultura (Dias et al., 2003; Dutra, 2003; Paula Junior \& Zambolim, 1998) ou mesmo utilizando-se de controle químico, com nematicidas aplicados diretamente no solo. Esses métodos nem sempre são eficientes; além disso, os produtos químicos podem trazer riscos ao meio ambiente, tanto pela contaminação das águas como pelos resíduos que deixam no próprio solo, além de não serem seletivos, afetando toda a biótica do solo. Como a alface é uma cultura de ciclo curto, resíduos podem ser encontrados também no produto comercial, acarretando riscos para a alimentação humana.

O uso de cultivares resistentes é o método mais viável para o controle de nematoides fitoparasitas (Kanayama et al., 2009; Ferreira et al., 2010), não elevando o custo de produção, exceto pela compra da própria semente. No entanto, nem sempre é possível, pela falta de cultivares resistentes que atendam às exigências do mercado.

A identificação de fontes de resistência aos nematoides das galhas Meloidogyne spp. em alface, preferencialmente entre cultivares comerciais, bem como a busca pelo desenvolvimento de cultivares resistentes, adaptadas às diversas condições brasileiras, têm sido preocupação de alguns pesquisadores, especialmente a partir do início da década de 1990 (Fiorini et al., 2007; Silva et al., 2008).

Charchar (1991) observou que, em condições de campo, as cultivares do tipo lisa, quando compradas com as do tipo crespa, são mais afetadas por nematoides das galhas, principalmente quando cultivadas em solos infestados em épocas de temperaturas e umidades do solo mais elevadas.

Trabalhando em solo naturalmente infestado com mistura de $M$. incognita raça 1 e $M$. javanica, Charchar \& Moita (1996) observaram que, dentre os materiais avaliados, algumas cultivares apresentaram maiores níveis de resistência a esses patógenos. Entre essas, sobressaíram-se algumas cultvares de folhas crespas, com destaque para Grand Rapids (TRB), Grand Rapids e A. Salinas. Gomes et al. (2002) verificaram que as cultivares do grupo de folhas crespas repolhuda Salinas 88, Lorca e Legacy apresentaram resistência a $M$. incognita, podendo constituir importantes fontes de resistência para serem utilizadas em programas de melhoramento.

Com os estudos de herança para o caráter resistência aos nematoides das galhas Meloidogyne incognita realizados a partir do cruzamento entre as cultivares Regina 71 (suscetível) e Grand Rapids (resistente), evidenciou-se que, o controle genético é feito por um único loco gênico (Gomes et al., 2000). Neste estudo também observou-se que o gene que controla o caráter apresenta efeito predominantemente aditivo, com herdabilidade no sentido amplo relativamente alta, o que facilita a seleção de genótipos resistentes.

Objetivou-se, neste trabalho, caracterizar linhagens avançadas de alface oriundas de alface de folhas lisas, oriundas do cruzamento [('Regina 71' x 'Grand Rapids') x 'Elisa'] quanto à resistência à $M$. javanica, visando à seleção daquelas homozigotas para o caráter.

\section{MATERIAL E MÉTODOS}

Realizou-se, o experimento em estufa, no período de fevereiro a junho de 2008, nas dependências da HortiAgro Sementes Ltda., no município de Ijaci, localizado

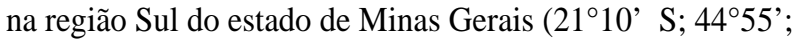
$832 \mathrm{~m}$ de altitude). A temperatura média anual foi de $19,4^{\circ} \mathrm{C}$, com médias mínimas de $14,8^{\circ} \mathrm{C}$ e máximas de $26,1^{\circ} \mathrm{C}$. O delineamento experimental utilizado foi o de blocos casualizados, com quatro repetições e oito plantas por parcela experimental.

Os tratamentos foram compostos pelas cultivares parentais Regina 71 e Grand Rapids, cinco linhagens $\mathrm{F}_{4} \mathrm{e}$ seis linhagens $\mathrm{F}_{5}$. A cultivar Regina 71 caracteriza-se por apresentar folhas soltas e lisas, tolerância ao florescimento prematuro e suscetibilidade à $M$. incognita raça 1 ; Grand Rapids é do tipo crespa de folhas soltas e apresenta resistência à $M$. incognita e $M$. javanica (Gomes et al., 2000, 2002) e suscetibilidade ao florescimento precoce.

As linhagens $\mathrm{F}_{4}$ foram obtidas a partir do cruzamento entre as cultivares Regina 71, Grand Rapids, com um retrocruzamento para a cultivar de folhas lisas Elisa. A população $F_{1}$ originou-se do cruzamento entre 'Regina 71' e 'Grand Rapids'. Depois $F_{1}$ foi autofecundada, obtendo-se a população $F_{2}$. Seguindo o método de melhoramento pedigree (Borém \& Miranda, 2005), avaliou- 
se a população $\mathrm{F}_{2}$ quanto à resistência à $M$. incognita $\mathrm{spp}$. e tolerância ao florescimento prematuro. As progênies selecionadas deram origem às linhagens $\mathrm{F}_{3}$, sendo as plantas dessas linhagens avaliadas quanto ao tipo de folha. Progênies $\mathrm{F}_{3}$ selecionadas para tipo de folhas lisas que foram retrocruzadas com a cultivar Elisa, obtendo-se linhagens $\mathrm{F}_{4}$, que foram utilizadas no presente trabalho. Algumas progênies selecionadas a partir das linhagens $F_{4}$ foram autofecundadas, obtendo-se linhagens $\mathrm{F}_{5}$, também utilizadas nesse estudo.

Foram utilizadas cinco linhagens $\mathrm{F}_{4}$, denominadas AFX 020B 006 13, AFX 020B 006 20, AFX 022B 010 14, AFX 022B 01017 e AFX 022B 010 31; e seis linhagens $F_{5}$, denominadas AFX 018C 00223 15, AFX 018C 00223 22, AFX $018 C 00242$ 16, AFX $018 \mathrm{C} 00542$ 20, AFX $018 \mathrm{C} 00544$ 22 e AFX $018 \mathrm{C} 0054428$.

A semeadura foi realizada em bandejas de poliestireno expandido de 128 células, contendo substrato comercial Plantmax ${ }^{\circledR}$, semeando-se duas a três sementes por célula. Após a germinação e emergência, quando as plântulas apresentavam o estádio de primeira folha definitiva, procedeu-se ao desbaste, deixando apenas uma plântula em cada célula. Vinte e seis dias após a semeadura, as mudas foram infestadas com ovos de $M$. javanica, na concentração de 1200 ovos célula ${ }^{-1}$, correspondendo a 30 ovos $\mathrm{cm}^{-3}$ de substrato, o que correspondeu à população inicial de nematoides $\left(\mathrm{P}_{\mathrm{i}}\right)$. A extração de ovos e infestação do substrato ocorreu conforme a técnica de Hussey \& Barker (1973), modificada por Boneti \& Ferraz (1981).

Utilizaram-se também, em cada bandeja, oito plantas de tomateiro (Solanun lycopersicum L.) cultivar Santa Clara, padrão de suscetibilidade aos nematoides das galhas, as quais serviram no momento da avaliação para se verificar a eficiência e o grau de infestação do inóculo, visualizando a presença de galhas em suas raízes.

As plantas permaneceram nas bandejas, em casa de vegetação, até quarenta e sete dias após a inoculação dos nematoides, quando foram feitas as avaliações. Nessa época, verificou-se alta incidência de galhas e massas de ovos nas raízes das plantas de tomateiro. Cada planta foi avaliada individualmente para as características índice de galhas, número de ovos, fator de reprodução e índice de reprodução.

Para o caráter índice de galhas, a avaliação foi feita visualmente, mediante critério de notas para as raízes das plantas ainda com substrato variando de 1 a 5 (nota 1: poucas galhas visíveis $(<10$ galhas $)$, pequenas $(<1 \mathrm{~mm})$; nota 2: poucas galhas visíveis, com tamanho intermediário (1 a $3 \mathrm{~mm}$ ); nota 3: número intermediário de galhas visíveis (10-30 galhas), de tamanho intermediário e algumas grandes (>3 mm); nota 4: muitas galhas visíveis (> 30 galhas), predominando as galhas grandes (>3 mm), com poucas de tamanho intermediário e algumas coalescentes; nota 5: torrão com muitas galhas visíveis (> 30 galhas), grandes e elevado número de galhas coalescentes (Fiorini et al., 2007).

Em seguida cada planta teve suas raízes lavadas com cuidado, evitando-se o jato d'água direto em suas raízes, retirando-se o excesso de substrato. As raízes limpas foram secas com papel toalha e pesadas, obtendo-se a massa fresca de raiz em gramas.

Para a avaliação do número de ovos, procedeu-se à extração conforme técnica de Hussey \& Barker (1973), modificada por Bonetti \& Ferraz (1981). Os ovos foram contados em microscópio estereoscópio, obtendo-se o número de ovos por planta de alface (NOA) e tomate (NOT), os quais corresponderam à população final $\left(\mathrm{P}_{\mathrm{f}}\right)$ de nematoides. De posse desses dados, foram obtidos os caracteres Fator de Reprodução (FR) e Índice de Reprodução (IR). O Fator de Reprodução corresponde à razão entre a população final e inicial de nematoides $(\mathrm{Fr}=$ $\left.\mathrm{P}_{\mathrm{f}} / \mathrm{P}_{\mathrm{i}}\right)$ e o Índice de Reprodução à razão entre o número de ovos no sistema radicular da alface e o número de ovos no sistema radicular do tomateiro em cada bloco, multiplicado por $100($ IR = NOA/NOT $x 100)$.

As análises foram realizadas com o auxílio do programa SAS (Statistical Analysis System Institute - SAS Institute, 1999). Comparou-se separadamente a média de cada testemunha, 'Regina 71' (suscetível) e 'Grand Rapids' (resistente), com a média de cada linhagem avançada, utilizando-se o teste de Dunnett (5\%). A significância entre a linhagem e a cultivar Grand Rapids (resistente) e a não significância em relação à Regina 71 (suscetível), para todos os caracteres, identifica a linhagem como homozigota resistente. Por outro lado, a significância entre a linhagem e cultivar Regina 71 e a não significância a Grand Rapids para todos os caracteres, caracteriza a linhagem como homozigota suscetível. Qualquer condição divergente das citadas acima caracteriza a linhagem como segregante.

\section{RESULTADOS E DISCUSSÃO}

Ao comparar as 11 linhagens avançadas [('Regina 71' x 'Grand Rapids') x 'Elisa'] com as testemunhas resistente ('Grand Rapids') e suscetível ('Regina 71'), verificou-se que para índice de galhas (ING) (Tabela 1), sete linhagens avançadas (AFX 018C 00223 15, AFX 018C 00223 22, AFX $018 \mathrm{C} 00542$ 20, AFX $018 \mathrm{C} 00544$ 22, AFX 018C 00544 28, AFX 022B 01014 e AFX 022B 010 31) não diferiram significativamente da cultivar resistente Grand 
Rapids, diferindo no entanto da cultivar suscetível Regina 71 , sendo, portanto, consideradas homozigotas resistentes para a característica resistência ao nematoide das galhas M. javanica. As outras quatro linhagens avançadas (AFX 018C 00242 16, AFX 020B 006 13, AFX 020B 00620 e AFX 022B 010 17) diferiram tanto de 'Grand Rapids' quanto de 'Regina 71', sendo, portanto segregantes para tal característica.

Considerando-se os caracteres número de ovos e fator de reprodução, sete linhagens avançadas (AFX 018C 00223 15, AFX 018C 00223 22, AFX 018C 00242 16, AFX 018C 00542 20, AFX 018C 00544 22, AFX 018C 0054428 e AFX 022B 010 31) não diferiram da cultivar Grand Rapids para ambas as características, diferindo no entanto da cultivar suscetível Regina 71, sendo consideradas homozigotas resistentes. Quatro linhagens avançadas (AFX 020B 006 13, AFX 020B 006 20, AFX 022B 01014 e AFX 022B 010 17) diferiram tanto de 'Grand Rapids' quanto de 'Regina 71', portanto segregantes (Tabelas 2 e 3).

Observou-se que, ao considerar o caráter índice de reprodução, oito linhagens avançadas (AFX 018C 00223 15, AFX 018C 00223 22, AFX $018 \mathrm{C} 00242$ 16, AFX 018C 005 42 20, AFX 018C 00544 22, AFX $018 \mathrm{C} 00544$ 28, AFX 022B 01017 e AFX 022B 010 31) não diferiram significativamente da cultivar resistente Grand Rapids, porém diferiram da cultivar suscetível Regina 71, sendo consideradas homozigotas resistentes. Nesse caso, apenas três linhagens avançadas (AFX 020B 006 13, AFX 020B 00620 e AFX 022B 010 14) diferiram tanto de uma quanto da outra cultivar, sendo consideradas segregantes (Tabela 4).

Verificou-se que seis linhagens avançadas (AFX 018C 00223 15, AFX 018C 00223 22, AFX $018 \mathrm{C} 00542$ 20, AFX 018C 00544 22, AFX 018C 0054428 e AFX 022B 010 31) se mostraram como homozigotas resistentes para todas as quatro características avaliadas e apenas duas linhagens avançadas (AFX 020B 00613 e AFX 020B 006 20) foram consideradas segregantes para as mesmas características.

Resultados semelhantes foram obtidos por Fiorini et al. (2007), trabalhando com 39 famílias $F_{2: 3}$ de alface, sendo evidenciadas a existência de 10 famílias homozigotas para resistência aos nematoides das galhas Meloidogyne spp.

A linhagem avançada AFX 018C 0024216 foi considerada segregante apenas para índice visual de galhas; entretanto para número de ovos por sistema radicular, fator de reprodução e índice de reprodução apresentou-se como homozigota resistente. Já a linhagem avançada AFX 022B 01014 reve comportamento contrário, sendo caracterizada como homozigota resistente apenas para índice visual de galhas e segregante para número de ovos por sistema radicular, fator de reprodução e índice de reprodução.

Tabela 1 - Comparação das médias das notas para índice visual de galhas (ING) de 11 linhagens avançadas de alface do cruzamento [('Regina 71' x 'Grand Rapids') x 'Elisa'] inoculadas com ovos de M. javanica. Lavras, UFLA, 2008.

\begin{tabular}{ccccc}
\hline \multirow{2}{*}{ Tratamento } & Média nota & \multicolumn{2}{c}{ Probabilidade } & Reação \\
\cline { 3 - 4 } & & T>Grand Rapids & T<Regina & Homozigota/Resistente \\
AFX018C0022315 & 1,190 & $\mathrm{~ns}$ & $*$ & Homozigota/Resistente \\
AFX018C0022322 & 1,000 & $\mathrm{~ns}$ & $*$ & Segregante \\
AFX018C0024216 & 1,845 & $*$ & $*$ & Homozigota/Resistente \\
AFX018C0054220 & 1,377 & $\mathrm{~ns}$ & $*$ & Homozigota/Resistente \\
AFX018C0054422 & 1,000 & $\mathrm{~ns}$ & $*$ & Homozigota/Resistente \\
AFX018C0054428 & 1,095 & $\mathrm{~ns}$ & $*$ & Segregante \\
AFX020B00613 & 2,035 & $*$ & $*$ & Segregante \\
AFX020B00620 & 1,877 & $*$ & $*$ & Homozigota/Resistente \\
AFX022B01014 & 1,627 & $\mathrm{~ns}$ & $*$ & Segregante \\
AFX022B01017 & 1,907 & $*$ & & Homozigota/Resistente \\
AFX022B01031 & 1,600 & $\mathrm{~ns}$ & & \\
Grand Rapids & 1,065 & & & \\
Regina 71 & 3,470 & & &
\end{tabular}


A linhagem avançada AFX 022B 01017 foi reprodução e segregante para índice visual de galhas, considerada homozigota resistente apenas para índice de número de ovos por sistema radicular e fator de reprodução.

Tabela 2 - Comparação das médias do número de ovos/sistema radicular de 11 linhagens avançadas de alface do cruzamento [('Regina 71' x 'Grand Rapids') x 'Elisa'] inoculadas com ovos de M. javanica. Lavras, UFLA, 2008.

\begin{tabular}{ccccc}
\hline & \multicolumn{5}{c}{ Probabilidade } \\
\hline Tratamento & Média ovos & T> Grand Rapids & T< Regina & Reação \\
\hline AFX018C0022315 & 237,510 & $\mathrm{~ns}$ & $*$ & Homozigota/Resistente \\
AFX018C0022322 & 184,375 & $\mathrm{~ns}$ & $*$ & Homozigota/Resistente \\
AFX018C0054220 & 2716,187 & $\mathrm{~ns}$ & $\mathrm{~ns}$ & Homozigota/Resistente \\
AFX018C0054422 & 274,127 & $\mathrm{~ns}$ & $*$ & Homozigota/Resistente \\
AFX018C0054428 & 686,625 & $\mathrm{~ns}$ & $*$ & Homozigota/Resistente \\
AFX020B00613 & 4837,187 & $*$ & $*$ & Homozigota/Resistente \\
AFX020B00620 & 4489,187 & $*$ & $*$ & Segregante \\
AFX022B01014 & 4398,625 & $*$ & $*$ & Segregante \\
AFX022B01017 & 3410,720 & $*$ & $*$ & Segregante \\
AFX022B01031 & 2879,045 & $\mathrm{~ns}$ & & Homozigota/Resistente \\
Grand Rapids & 413,125 & & & \\
Regina 71 & 7916,365 & & & $*$
\end{tabular}

$\mathrm{T}=$ Probabilidade das médias dos tratamentos serem diferentes das médias das testemunhas.

*Teste de Dunnett, a 5\% de probabilidade.

Tabela 3 - Comparação das médias do Fator de Reprodução (FR) de 11 linhagens avançadas de alface do cruzamento [('Regina 71' x 'Grand Rapids') x 'Elisa'] inoculadas com ovos de M. javanica. Lavras, UFLA, 2008.

\begin{tabular}{ccccc}
\hline & & \multicolumn{2}{c}{ Probabilidade } & \\
\cline { 3 - 4 } Tratamento & Média do FR & T> Grand Rapids & T< Regina & Reação \\
\hline AFX018C0022315 & 0,195 & $\mathrm{~ns}$ & $*$ & Homozigota/Resistente \\
AFX018C0022322 & 0,152 & $\mathrm{~ns}$ & $*$ & Homozigota/Resistente \\
AFX018C0024216 & 1,542 & $\mathrm{~ns}$ & $*$ & Homozigota/Resistente \\
AFX018C0054220 & 2,262 & $\mathrm{~ns}$ & $*$ & Homozigota/Resistente \\
AFX018C0054422 & 0,230 & $\mathrm{~ns}$ & $*$ & Homozigota/Resistente \\
AFX018C0054428 & 0,572 & $\mathrm{~ns}$ & $*$ & Homozigota/Resistente \\
AFX020B00613 & 4,030 & $*$ & $*$ & Segregante \\
AFX020B00620 & 3,740 & $*$ & $*$ & Segregante \\
AFX022B01014 & 3,665 & $*$ & $*$ & Segregante \\
AFX022B01017 & 2,845 & $*$ & $*$ & Segregante \\
AFX022B01031 & 2,400 & $\mathrm{~ns}$ & & \\
Grand Rapids & 0,342 & & & \\
Regina 71 & 6,597 & & $*$ Hota/Resistente
\end{tabular}

$\mathrm{T}=$ Probabilidade das médias dos tratamentos serem diferentes das médias das testemunhas.

*Teste de Dunnett, a 5\% de probabilidade. 
Tabela 4 - Comparação das médias do Índice de Reprodução (IR) de 11 linhagens avançadas de alface do cruzamento [('Regina 71' x 'Grand Rapids') x 'Elisa'] inoculadas com ovos de M. javanica. Lavras, UFLA, 2008.

\begin{tabular}{ccccc}
\hline & & \multicolumn{2}{c}{ Probabilidade } & \\
\cline { 3 - 4 } Tratamento & Média do IR & T> Grand Rapids & T< Regina & Reação \\
\hline AFX018C0022315 & 1,217 & $\mathrm{~ns}$ & $*$ & Homozigota/Resistente \\
AFX018C0022322 & 0,990 & $\mathrm{~ns}$ & $*$ & Homozigota/Resistente \\
AFX018C0024216 & 11,190 & $\mathrm{~ns}$ & $*$ & Homozigota/Resistente \\
AFX018C0054220 & 14,270 & $\mathrm{~ns}$ & $*$ & Homozigota/Resistente \\
AFX018C0054422 & 1,325 & $\mathrm{~ns}$ & $*$ & Homozigota/Resistente \\
AFX018C0054428 & 3,465 & $\mathrm{~ns}$ & $*$ & Homozigota/Resistente \\
AFX020B00613 & 26,712 & $*$ & $*$ & Segregante \\
AFX020B00620 & 24,392 & $*$ & $*$ & Segregante \\
AFX022B01014 & 23,262 & $*$ & $*$ & Segregante \\
AFX022B01017 & 18,302 & $\mathrm{~ns}$ & $*$ & Homozigota/Resistente \\
AFX022B01031 & 14,435 & $\mathrm{~ns}$ & & Homozigota/Resistente \\
Grand Rapids & 2,247 & & & \\
Regina 71 & 46,297 & & $*$ & \\
\hline
\end{tabular}

$\mathrm{T}$ = Probabilidade das médias dos tratamentos serem diferentes das médias das testemunhas.

*Teste de Dunnett, a 5\% de probabilidade.

Embora o índice visual de galhas seja um critério subjetivo, visto que a avaliação é visual, mediante uma nota atribuída pelo avaliador, apresenta-se como um método de grande interesse para o melhoramento da alface, para resistência dos nematoides das galhas, pois permite a preservação da planta selecionada e colheita de suas sementes, dando continuidade ao processo de melhoramento.

A maioria das linhagens $\mathrm{F}_{4}$, que já haviam sido previamente avaliadas para o caráter índice visual de galhas, foram consideradas como segregantes para as outras características avaliadas, enquanto as linhagens $\mathrm{F}_{5}$, por estar em uma geração à frente, estando mais avançadas no programa de melhoramento, foram consideradas como homozigotas resistentes, em sua maioria, para as quatro características avaliadas, mostrando que a seleção feita em $\mathrm{F}_{4}$ foi eficiente e que o cruzamento com a cultivar Elisa não interferiu na expressão da resistência ao nematoide das galhas $M$. javanica.

A resistência à $M$. javanica foi confirmada em seis linhagens, para todas características estudadas. Em trabalhos futuros deve ser estudada a possibilidade de que as linhagens aqui estudadas possam ser também resistentes a outras espécies de Meloidogyne spp., conforme estudos já realizados por Gomes et al. (2000) e Carvalho Filho et al. (2008), que também identificaram resistência monogênica para $M$. incognita. Esses resultados eram esperados, já que as plantas que deram origem a essas linhagens haviam sido selecionadas anteriormente para a resistência a Meloidogyne spp. No estudo realizado por Gomes et al. (2000), observou-se que mesmo com a mistura de raças de $M$. incognita o comportamento quanto à resistência foi o mesmo; assim os autores sugeriram que talvez o mesmo gene controle a resistência a todas as raças. Contudo, serão necessárias novas avaliações com a espécie/raças de $M$. incognita raças 1, 2, 3 e 4 para confirmação dessas evidências para as linhagens $\mathrm{F}_{4}$ e $\mathrm{F}_{5}$ aqui testadas. Essa comprovação é conveniente, visto que essas populações, $M$. incognita, são endêmicas de regiões semelhantes e podem se desenvolver na mesma área (Tihohod, 2000).

As linhagens AFX 018C 00223 15, AFX 018C 002 23 22, AFX $018 \mathrm{C} 00542$ 20, AFX 018C 00544 22, AFX0 18C 0054428 e AFX 022B 010 31, poderão ser utilizadas como novas fontes de resistência à $M$. javanica em programas de melhoramento de alface, além da possibilidade de serem utilizadas em avaliações junto a produtores, para obtenção de novas cultivares de alface de folhas lisas, tolerantes ao florescimento prematuro.

\section{CONCLUSÕES}

Foram encontradas seis linhagens (AFX 018C 002 23 15, AFX 018C 00223 22, AFX 018C 00542 20, AFX 018C 
00544 22, AFX $018 C 0054428$ e AFX 022B 010 31) de alface que poderão ser utilizadas em programas de melhoramento, como fontes de resistência à Meloidogyne javanica.

\section{AGRADECIMENTOS}

À CAPES, pela concessão da bolsa de doutorado à primeira autora e pelo auxílio financeiro na condução dos experimentos. À FAPEMIG, FAEPE, CNPq, HortiAgro Sementes Ltda. e UFLA.

\section{REFERÊNCIAS BIBLIOGRÁFICAS}

BONETI, J.I.S.; FERRAZ, S. Modificação do método de Hussey \& Barker para extração de ovos de Meloidogyne exigua de raízes de cafeeiro. Fitopatologia Brasileira, Brasília, v.6, p.553, 1981.

BORÉM, A.; MIRANDA, G.V. Melhoramento de plantas. 4.ed. Viçosa, MG: UFV, 2005. 525p.

CAMPOS, V.P. Doenças causadas por nematoides. Informe Agropecuário, Belo Horizonte, v.11, n.172, p.2128, 1985.

CAMPOS, V.P.; CAMPOS, J.R.; SILVA, L.H.C.P.; DUTRA, M.R. Manejo de nematoides em hortaliças. In: SILVA, L.H.C.P.; CAMPOS, J.R.; NOJOSA (Eds.). Manejo integrado: doenças e pragas em hortaliças. Lavras: UFLA, 2001. p.125-158.

CARVALHO FILHO, J.L.S. de; GOMES, L.A.A.; WESTERICH, J.N.; MALUF, W.R.; CAMPOS, V.P.; FERREIRA, S. Inheritance of resistance of 'Salinas 88' lettuce to the root-knot nematode Meloidogyne incognita (Kofoid \& White) Chitwood. Revista Brasileira de Agrociência, Pelotas, v.14 n.2, abr./jun. 2008. Disponível em: <http://www.ufpel.tche.br/faem/agrociencia/v14n2h lartigo09.htm $>$. Acesso em: 28 set. 2010.

CHARCHAR, J.M. Comportamento de cultivares de alface à infecção por nematoides de galhas. Horticultura Brasileira, Brasília, v.9, p.35, 1991. Resumo.

CHARCHAR, J.M.; MOITA, A.W. Reação de cultivares de alface à infecção por misturas populacionais de Meloidogyne incognita raça 1 e Meloidogyne javanica em condições de campo. Horticultura Brasileira, Brasília, v.14, n.2, p.185-189, 1996.

DIAS, W.P.; ASMUS, G.L.; CARNEIRO, G.E. de S. Manejo integrado de nematoides na cultura da soja.
Fitopatologia Brasileira, Brasília, v.28, p.30-33, 2003. Suplemento.

DUTRA, M.R.; CAMPOS, V.P. Manejo do solo e da irrigação como nova tática de controle de Meloidogyne incognita em feijoeiro. Fitopatologia Brasileira, Brasília, v.28, p.608-614, 2003.

FERREIRA, S.; GOMES, L.A.A.; MALUF, W.R.; CAMPOS, V.P.; CARVALHO FILHO, J.L.S. de; SANTOS, D.C. Resistance of dry bean and snap bean cultivars to root-knot nematodes. HortScience, Amsterdam, v.45, n.2, p.320-322, 2010.

FIORINI, C.V.A.; GOMES, L.A.A.; LIBÂNIO, R.A.; MALUF, W.R.; CAMPOS, V.P.; LICURSI, V.; MORETTO, P.; SOUZA, L.A.; FIORINI, I.V.A. Identificação de famílias $\mathrm{F}_{2: 3}$ de alface homozigotas resistentes aos nematoides das galhas.

Horticulturua Brasileira, Braslília, v.25, p.509-513, 2007.

FREITAS, L.G. O controle biológico dentro do contexto de manejo integrado de nematoides. Fitopatologia Brasileira, Brasília, v.28, p.24-29, 2003. Suplemento.

GOMES, L.A.A.; MALUF, W.R.; AZEVEDO, S.M.; FREITAS, J.A.; LICURSI, V. Reação de cultivares de alface a infecção por Meloidogyne javanica.

Horticultura Brasileira, Brasíllia, v.14, n.1, p.99, 2002.

GOMES, L.A.A.; MALUF, W.R.; CAMPOS, V.P. Inherintance of the resistance reaction of the lettuce cultivar 'Grand Rapids' to the southern root-knot nematode Meloidogyne incognita (Kofoid \& White) Chitwood. Euphytica, Alexandria, v.114, n.1, p.37-46, 2000.

HUSSEY, R.S.; BARKER, K.R. A comparison of methods collecting inocula of Meloidogyne spp. Including a new technique. Plant Disease Report, Saint Paul, v.57, n.12, p.1025-1028, 1973.

KANAYAMA, F.S.; SERA, G.H.; SERA, T.; MATA, J.S. da; RUAS, P.M.; ITO, D.S. Progênies de Coffea arabica cv. IPR 100 com resistência ao nematoide Meloidogyne incognita raça 1. Ciência e Agrotecnologia, Lavras, v.33, n.5, p.1321-1326, set./out. 2009. 
LOPES, C.A.; SANTOS, J.R.M. dos. Doenças do tomateiro. Brasília: EMBRAPA-CNPH/EMBRAPA-SPI, 1994. 61p.

PAULA JUNIOR, T.J. de; ZAMBOLIM, L. Doenças. In: VIEIRA, C.; PAULA JUNIOR, T.J.; BORÉM, A. (Eds.). Feijão: aspectos gerais e cultura no Estado de Minas Gerais. Viçosa, MG: UFV, 1998. p.373-433.

SANTOS, H.S.; SOUZA, R.J. Efeito de métodos de plantio e manejo do solo infestado com Meloidogyne javanica na produção de alface sob estufa plástica. Horticultura Brasileira, Botucatu, v.14, n.1, p.19-22, 1996.
SILVA, R.R.; GOMES, L.A.A.; MONTEIRO, A.B.; MALUF, W.R.; CARVALHO FILHO, J.L.S. de; MASSAROTO, J.A. Linhagens de alface-crespa para o verão resistentes ao Meloidogyne javanica e ao vírus mosaico-da-alface. Pesquisa Agropecuária Brasileira, Brasília, v.43, n.10, p.1349-1356, 2008.

STATISTICAL ANALYSIS SYSTEM INSTITUTE.SAS user's guide statistics. Versão 8. Cary, 1999. 956p.

TIHOHOD, D. Nematologia agrícola aplicada. 2.ed. Jaboticabal: FUNEP, 2000. 473p. 